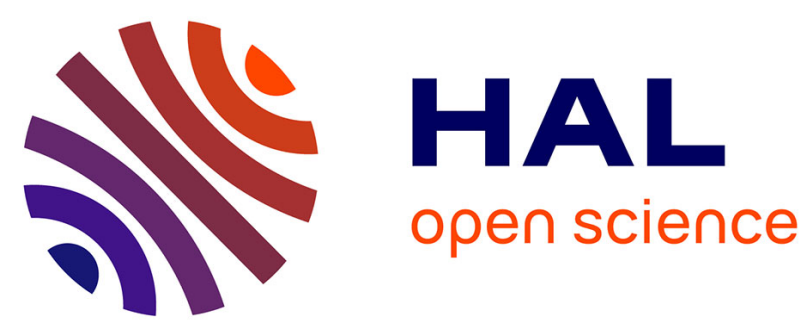

\title{
COEFFICIENT DE RÉFLEXION D'UN FAISCEAU BORNÉ SUR UNE PLAQUE
}

I. Molinero, M. de Billy, G. Quentin, O. Leroy, A. Vinckier

\section{To cite this version:}

I. Molinero, M. de Billy, G. Quentin, O. Leroy, A. Vinckier. COEFFICIENT DE RÉFLEXION D'UN FAISCEAU BORNÉ SUR UNE PLAQUE. Journal de Physique Colloques, 1990, 51 (C2), pp.C2-1281C2-1284. 10.1051/jphyscol:19902301 . jpa-00230643

\section{HAL Id: jpa-00230643 https://hal.science/jpa-00230643}

Submitted on 1 Jan 1990

HAL is a multi-disciplinary open access archive for the deposit and dissemination of scientific research documents, whether they are published or not. The documents may come from teaching and research institutions in France or abroad, or from public or private research centers.
L'archive ouverte pluridisciplinaire HAL, est destinée au dépôt et à la diffusion de documents scientifiques de niveau recherche, publiés ou non, émanant des établissements d'enseignement et de recherche français ou étrangers, des laboratoires publics ou privés. 
ler Congrès Français d'Acoustique 1990

\title{
COEFFICIENT DE REFLEXION D'UN FAISCEAU BORNE SUR UNE PLAQUE
}

\author{
I. MOLINERO $(1)$, M. DE BILLY, G. QUENTIN, O. LEROY* et A. VINCKIER* \\ Université Paris 7, Groupe de Physique des solides, Tour 23, 2 Place \\ Jussieu, F-75251 Par1s Cedex 05, France \\ ${ }^{*}$ K.U.L.C.K., E. Sabbelaan, B-8500 Kortrijk, Belgique
}

\begin{abstract}
Résumé - Le coefficient de reflexion d'un faisceau acoustique bomé, incident sur une plaque est calculé à l'aide d'un nouveau modèle mathématique qui décrit le faisceau en termes d'ondes planes inhomogènes. Les courbes théoriques obtenues numériquement sont comparées ayec les mesures expérimentales. Un bon accord est observé en ce qui concerne l'existence et la position des minima.
\end{abstract}

\begin{abstract}
The bounded beam reflection coefficient for plates is described using a new mathematical model. This model uses a simple representation of the incident beam by inhomogeneous infinite plane waves. The numerical calculations are compared with experimental data. The agreement is good for the existence and the angular positions of the minima.
\end{abstract}

\section{1 - INTRODUCTION - POSITION DU PROBLEME}

Les mesures du coefficient de réflexion réalisées à l'aide d'un faisceau acoustique borné, incident sur une plaque immergée, révèlent l'existence de minima dont la profondeur est généralement différente de zéro /1/. Bien des auteurs ont décrit ce type de faisceau sur la base d'une analyse de Fourier du profil. Ainsi dans le modèle proposé par Ngoc et Mayer /2/, les auteurs considèrent une interférence entre les ondes planes se propageant dans des directions différentes. Nous montrons que suivant la précision avec laquelle sont effectués les calculs numériques du coefficient de réflexion sur la base de ce modèle d'ondes planes infinies homogènes, et en négligeant l'absorption, les minima tendent tous vers une valeur nulle. Ce résultat est illustré sur les figures 1-a et 1-b (le pas angulaire est divisé par un facteur 10 entre les deux courbes). Comme ce modèle n'est plus valable aux angles d'incidence élevés, nous proposons de décrire un faisceau borné au moyen d'une source finie d'ondes planes inhomogènes, toutes incidentes suivant le même angle.

\section{2- MODELE DES ONDES PLANES INHOMOGENES}

L'expression analytique d'une onde plane inhomogène se propageant dans la direction Oz est décrite par l'expression suivante $/ 3 /$ :

$$
A \exp (\beta x) \cdot \exp \left[i\left\{(\omega / v)^{2}+\beta^{2}\right\}^{1 / 2} \cdot z\right]
$$

Si $\phi(x, z)$ décrit la fonction potentielle du faisceau incident borné, on propose de décomposer cette fonction en ondes planes inhomogènes. On obtient alors :

$$
\phi(x, z)=\sum_{n=0}^{N} A_{n} \exp \left(\beta_{n} x\right) \cdot \exp \left[i\left\{(\omega / v)^{2}+\beta_{n}{ }^{2}\right\}^{1 / 2} \cdot z\right]
$$

où $N$ est un nombre entier fini. $A_{n}$ et $\beta_{n}$ sont des paramètres qui sont calculés à partir des conditions aux limites et qui dépendent de la largeur et de la forme du profil du faisceau incident. Pour $\mathrm{N}=60$, on obtient déjà une très bonne approximation.

$\mathrm{Si}$ nous introduisons maintenant le coefficient de réflexion d'ondes planes inhomogènes $/ 3 /$ pour chacune des ondes, nous avons la possibilités de recalculer le profil du faisceau réfléchi. En intégrant ce profil sur toute cette largeur et en divisant ce résultat par la quantité $\left(b_{1}-a_{1}\right)$ qui représente cette largeur de profil, nous obtenons, au niveau de l'interface $(z=0)$, une amplitude

adresse permanente : AEROSPATIALE (DCR/LM), 12 rue Pasteur, 92152 Suresnes, France. 
moyenne réfléchie $\boldsymbol{H}_{\mathrm{r}}$. En faisant de même pour le faisceau incident de largeur (b - a) nous obtenons une amplitude moyenne $\boldsymbol{H}_{\mathrm{j}}$. A partir de ces deux amplitudes, il est possible de définir le coefficient de réflexion pour un faisceau borné $R_{b}(\theta)$, incident suivant l'angle $\theta$, comme le rapport :

$$
R_{b}(\theta)=\boldsymbol{A}_{\mathrm{r}} / \mathbf{A}_{\mathrm{i}}=\left[\frac{\sum_{n=0}^{N} A_{n} R\left(\theta, \beta_{n}\right) \int_{a_{1}}^{b_{1}} \exp \left(\beta_{n} x\right) d x}{\sum_{n=0}^{N} A_{n} \int_{a}^{b} \exp \left(\beta_{n} x\right) d x}\right] \cdot(b-a) /\left(b_{1}-a_{1}\right)
$$

$R\left(\theta, \beta_{n}\right)$ est le coefficient de réflexion de l'onde plane inhomogène $n$,

[b - a] est l'intervalle d'intégration pour le faisceau incident,

$\left[b_{1}-a_{1}\right]$ est l'intervalle d'intégration pour le faisceau réfléchi.

En dehors des angles critiques, les faisceaux incidents et réfléchis ont la même largeur, $b-a=b_{1}-a_{1}$ et $R\left(\theta, \beta_{n}\right)$ devient égal au coefficient $R(\theta)$ défini pour des ondes planes infinies. Pour une incidence critique, le coefficient $R\left(\theta, \beta_{n}\right)$ devient plus compliqué et on doit tenir compte de l'ouverture effective du faisceau réfléchi $\left(b_{1}\right.$ - $\left.a_{1}\right)$. La figure 2-a montre la courbe $R_{b}(\theta)$ obtenue pour $a_{1} \approx a$ et $b_{1} \approx b$. On note que la profondeur des pics ne correspond pas a celle mesurée expérimentalement pour la même valeur de $\mathrm{Fd}=2,5 \mathrm{MHz} . \mathrm{mm}$ (cf. fig.4). Si nous considérons maintenant la largeur du faisceau réfléchi, nous obtenons un traçé (fig. 2-b) différent du précédent qui se rapproche davantage de la courbe expérimentale.

Notons que le coefficient de réflexion défini au paragraphe précédent peut être mesuré par voie optique. En effet, on montre /4/ que la diffraction de lumière au premier ordre par le faisceau réfléchi peut s'écrire :

$$
A_{1, \Gamma}=(\pi . L) / \lambda\left(b_{1}-a_{1}\right) \cdot \sum_{n=0}^{N} A_{n} R\left(\theta, \beta_{n}\right) \int_{a_{1}}^{b_{1}} \exp \left(\beta_{n} x\right) d x
$$

Pour le faisceau incident, on obtient :

$$
A_{1, i}=(\pi . L) / \lambda(b-a) \cdot \sum_{n=0}^{N} A_{n} \int_{a}^{b} \exp \left(\beta_{n} x\right) d x
$$

Le coefficient de réflexion s'exprime alors en fonction des amplitudes précédentes: $R_{b}(\theta)=A_{1, \mathrm{r}} / A_{1, i} \cdot$

\section{3- ETUDE EXPERIMENTALE}

Les mesures expérimentales de $R_{b}(\theta)$ ont été réalisées en mesurant les variations angulaires de I'amplitude du signal réfléchi spéculairement par une plaque de laiton immergée dans un réservoir rempli d'eau.

3.a - Dispositif expérimental

Nous avons utilisé un dispositif mécanique permettant des mesures en géométrie bistatique : l'émetteur est maintenu fixe, tandis que le récepteur et l'échantillon peuvent tourner indépendamment autour de l'axe $\mathrm{zz}^{\prime}$ (figure 3). L'encombrement dû aux supports des sondes limite la plage d'analyse angulaire de $8^{\circ}$ à $90^{\circ}$. Les deux capteurs ónt même diamètre et même fréquence centrale d'excitation. Une impulsion de $8 \mu \mathrm{s}$ de longueur excite le transducteur-émetteur. Deux fréquences ont été utilisées lors de nos mesures : $2 \mathrm{MHz}$ (diamètre de palpeur 12,7 mm) et $5 \mathrm{MHz}$ (diamètre de palpeur $6,35 \mathrm{~mm}$ ). Les diagrammes angulaires présentés dans cet article ne concernent que deux plaques de laiton d'épaisseur $d=1,25 \mathrm{~mm}$ et $\mathrm{d}=1,02 \mathrm{~mm}$.

\section{$3 . b$ - Résultats et discussion}

Sur les figures 4 et 5 , sont reproduites les variations expérimentales de l'amplitude du signal réfléchi spéculairement à une distance fixe $(14 \mathrm{~cm})$ de l'interface.

Sur le tableau 1 nous comparons les positions angulaires des minima mesurés expérimentalement et calculés par la théorie des ondes planes inhomogènes. On note un très bon accord entre les deux valeurs, l'écart étant inférieur à $2 \%$.

Pour $\mathrm{Fd}=2,5 \mathrm{MHz} \cdot \mathrm{mm}$, le rapport entre les profondeurs des minima correspondant aux modes $\mathrm{A}_{0}$ et $S_{0}$ sont respectés entre la théorie et l'expérience. Concernant le mode $A_{1}$, on note un certain désaccord qui pourrait s'expliquer par la non prise en compte de l'atténuation dans les calculs théoriques. Soulignons aussi la confirmation expérimentale de l'absence de minimum du coefficient de réflexion prévu théoriquement pour le mode $S_{1}$. Ce phénomène peut s'expliquer par l'annulation de la composante transversale du déplacement lorsque l'angle critique de Lamb 
est voisin de l'angle critique longitudinal $\theta_{\mathrm{LC}} / 1,5 /$.

Pour $\mathrm{Fd}=5,1 \mathrm{MHz} . \mathrm{mm}$, les modes $\mathrm{A}_{0}$ et $\mathrm{S}_{0}$ se comportent comme un seul mode, le mode de Rayleigh, ce qui peut expliquer le désaccord observé quant à la profondeur du minimum avec la théorie. Les comportements expérimentaux des minima relatifs aux autres modes $A_{1}, S_{1}, A_{2}$ et $A_{3}$ sont en bon accord avec les calculs théoriques. Le mode $S_{2}$ très peu visible sur la figure 5 a une trop faible réémission dans le fluide pour être observé expérimentalement. L'absence du mode $S_{2}$ est encore une conséquence du fait que $\theta_{\mathrm{LC}}=\theta_{\mathrm{S} 2}$.

\section{4- CONCLUSION}

Le bon accord observé entre les calculs théoriques et les mesures expérimentales du coefficient de réflexion d'une onde bornée sur une plaque libre immergée, valide le modèle proposé pour décrire un faisceau limité à partir d'ondes plancs inhomogènes.

Fd $=2,5$ MHz.mm

\begin{tabular}{lcc}
\multicolumn{3}{c}{$\mathrm{C}_{\mathrm{L}}=4370 \mathrm{~m} / \mathrm{s}, \mathrm{C}_{\mathrm{T}}=2170 \mathrm{~m} / \mathrm{s}$} \\
modes & $\theta_{\mathrm{LC}}=19,5^{\circ}$ & \\
expérience & théorie \\
$\mathrm{A}_{0}$ & $48,25^{\circ}$ & $47,6^{\circ}$ \\
$\mathrm{S}_{0}$ & $42,75^{\circ}$ & $43,5^{\circ}$ \\
$\mathrm{A}_{1}$ & $23,25^{\circ}$ & $23,2^{\circ}$ \\
$\mathrm{S}_{1}$ & &
\end{tabular}

$\mathbf{F d}=5,1 \quad$ MHz.mm

\begin{tabular}{lcl}
\multicolumn{3}{c}{$\mathrm{C}_{\mathrm{L}}=4636 \mathrm{~m} / \mathrm{s}, \mathrm{C}_{\mathrm{T}}=2147 \mathrm{~m} / \mathrm{s}$} \\
modes & $\theta_{\mathrm{LC}}=18,5^{\circ}$ \\
expérience & théorie \\
$\mathrm{A}_{0}-\mathrm{S}_{0}$ & $47^{\circ}$ & $47^{\circ}$ \\
$\mathrm{A}_{1}$ & $40^{\circ}$ & $40,1^{\circ}$ \\
$\mathrm{S}_{1}$ & $32^{\circ}$ & $32,5^{\circ}$ \\
$\mathrm{A}_{2}$ & $24^{\circ}$ & $24^{\circ}$ \\
$\mathrm{S}_{2}$ & - & $19,5^{\circ}$ \\
$\mathrm{S}_{3}$ & $16^{\circ}$ & $15,15^{\circ}$ \\
$\mathrm{A}_{3}$ & $11^{\circ}$ & $9,15^{\circ}$
\end{tabular}

Tableau 1 - Comparaison expérience/théorie des positions angulaires des minima ( - : modes non mis en évidence par le calcul et/ou l'expérience)

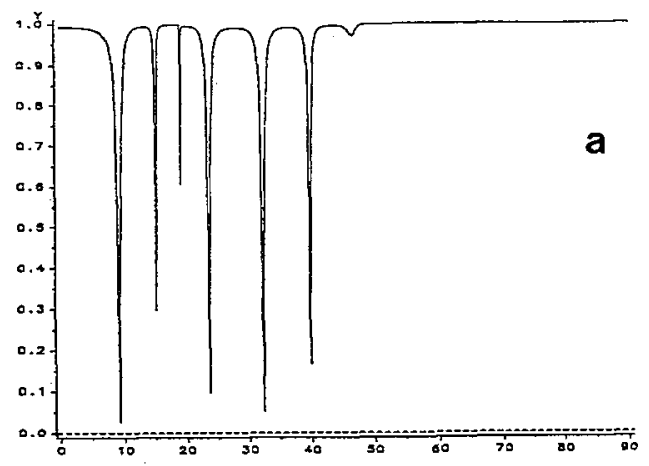

degrés

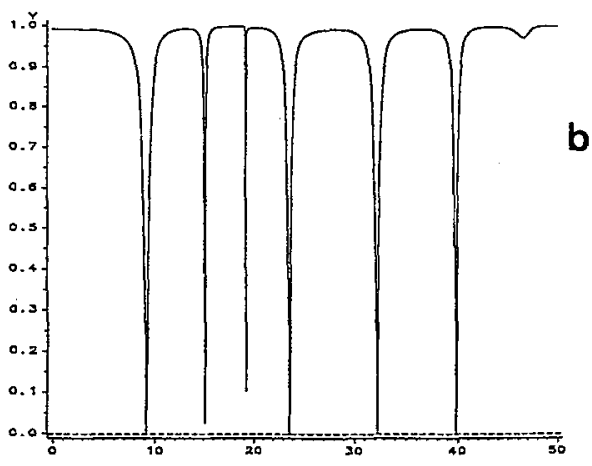

degrés

Fig. 1 - Courbes théoriques représentant le coefficient de réflexion pour une onde plane incidente $(\mathrm{F}=5 \mathrm{MHz}, \mathrm{d}=1,02 \mathrm{~mm})$ :

a- pas angulaire : 0,1 .

b- pas angulaire : 0,01 . 


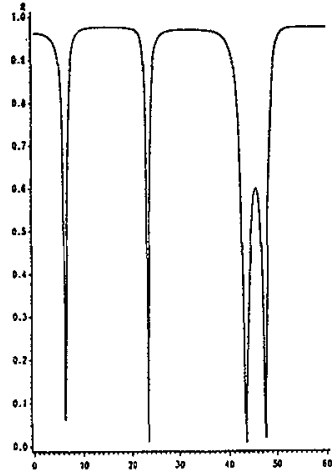

degrés

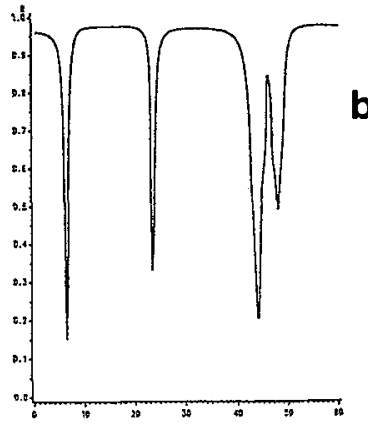

degrés

Fig. 2 - Courbes théoriques représentant le coefficient de réflexion pour une onde bornée incidente à partir du modèle des ondes planes inhomogènes : $a-(b-a)=\left(b_{1}-a_{1}\right)$.

b- $(b-a) \neq\left(b_{1}-a_{1}\right)$.

Fig. 3 - Géométrie de l'expérience.

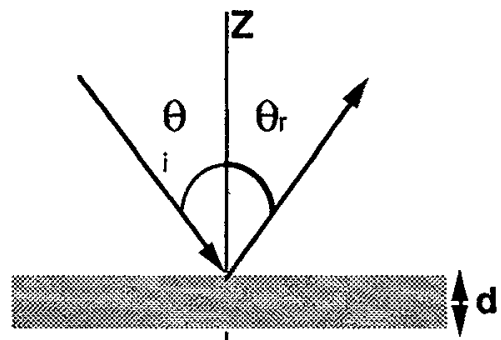

12

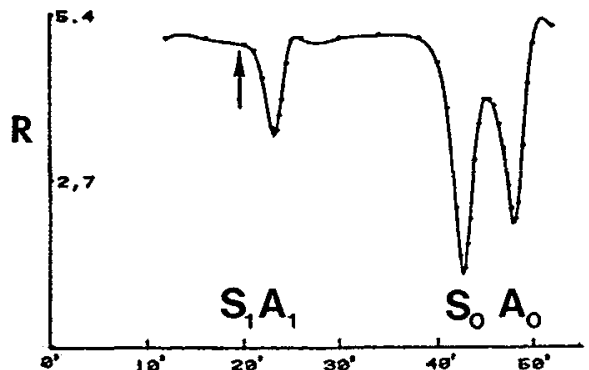

degrés

Fig. 4 - Courbe expérimentale de $R(\theta)$ pour $\mathrm{Fd}=2,5 \mathrm{MHz} \cdot \mathrm{mm}$

$(\longrightarrow$ : mode non mis en évidence)

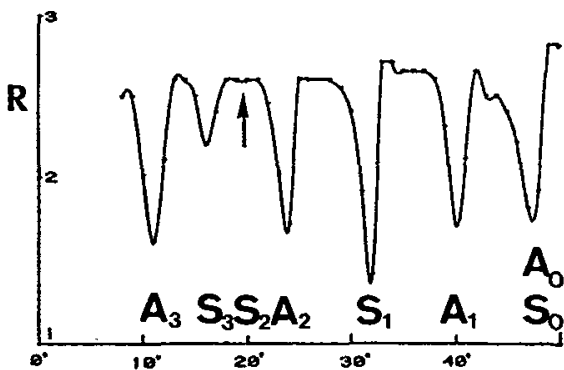

degrés

Fig. 5 - Courbe expérimentale de $R(\theta)$ pour $\mathrm{Fd}=5,1 \mathrm{MHz} . \mathrm{mm}$

$(\longrightarrow$ : mode non mis en évidence)

\section{REFERENCES}

/1/ Molinero, I., Thèse, Université Paris 7, 1987.

12/ Ngoc, T. D. K., Mayer, W.G., J. Acoust. Soc. Amer. 67 (1980) 1149.

13/ Claeys, J.M., Leroy, O., J. Acoust. Soc. Amer. 72 (1982) 585.

14/ Leroy, O., Claeys, J.M., J. Acoust. Soc. Amer. 75 (1984) 1346.

15/ Billy, M. de, Molinero, I., J. Acoust. Soc. Amer. $\underline{83}$ (1988) 1249. 